\title{
Tuberculosis Transmission in the Family
}

\author{
Pair Dong Wang ${ }^{\star 1}$ and R. S. Lin ${ }^{2}$ \\ ${ }^{1}$ Department of Internal Medicine, Taipei Municipal Chronic Disease Hospital, Taipei, Taiwan, \\ ${ }^{2}$ College of Public Health, National Taiwan University, Taipei, Taiwan
}

\begin{abstract}
Objective: To evaluate the risk of tuberculosis (TB) transmission from family members with infectious TB to other family members, and to examine whether household contact investigations had an impact on tuberculosis patterns.

Design: Under the direction of the Taipei Municipal Chronic Disease Hospital, 12 full-time public health nurses recruited the household contacts of TB patients. Chest X-ray examination was recommended for adult contacts. Child contacts received the Mantoux tuberculin skin test, and radiography was recommended if the results were positive. Setting: Family contacts of all TB index patients who attended one of 29 hospitals in Taipei, from July 1993 through June 1996. The medical records of index patients were obtained from the National Tuberculosis Registry.

Results: During the study period, the families of 3903 index patients, comprising 11873 contacts, were investigated. Among these, 4595 received radiography, for a response rate of $38.7 \%$. Of these, 284 had active pulmonary disease: $188(66.3 \%)$ had minimal disease, $79(27.8 \%)$ had moderately advanced disease, and only $17(5.9 \%)$ had far advanced disease. Overall, the index patients had more advanced TB: only 1261 (32.3\%) had minimal disease, while 2022 $(51.8 \%)$ had moderately advanced disease and 620 (15.9\%) had far advanced disease.

Conclusions: These data show a relatively high risk of intrafamily TB transmission. Our findings also show that family contact investigations may help to diagnose TB in earlier stages. Such an approach should greatly reduce the number of new TB cases and speed eradication of the disease.

(C) 2000 The British Infection Society
\end{abstract}

\section{Introduction}

Tuberculosis (TB) outbreaks often occur in overcrowded, poorly ventilated facilities when there is prolonged close exposure to an infectious person. Outbreaks of TB have been reported in a variety of crowded settings, including bars, ${ }^{1}$ hospitals, ${ }^{2}$ ships, ${ }^{3}$ commercial aircraft ${ }^{4}$ and school buses. ${ }^{5}$ Instances of widespread infection from a source case are not rare; indeed, that is what might be expected in homes where there is a person with active TB. Unfortunately, infected individuals are often lost in the crowd, because primary TB is usually asymptomatic and, without contact investigation, few people become aware that they have it. Numerous studies have found contact investigation to be a very efficient method to identify $\mathrm{TB}$ cases. ${ }^{6-9}$ In a contact study of 205 families in the U.S.A., Sue $^{6}$ found 326 of 2373 (13.7\%) contacts to have active TB. A similar study in Seoul, Korea, in 1959 found 357 (11.9\%) new cases of active TB among 3002 family contacts of index TB patients. ${ }^{7}$

\footnotetext{
* Please address all correspondence to: Pair Dong Wang, Taipei Municipal Chronic Disease Hospital, No. 530, Lin-Shan N. Road, Taipei, Taiwan. Accepted for publication 4 August 2000.
}

In Taiwan the magnitude of the TB problem is enormous. According to a recent estimate, approximately 15000 new cases of TB are identified annually in Taiwan: a crude incidence rate of 71.4 per 100000 population. Because each active case creates a pool that breeds TB and constitutes a great clinical and public health menace, an efficient method of contact investigation is needed to help decrease the speed of TB in Taiwan. In this prospective study we used household contact investigations to examine the risk of spread of TB from index patients to family members. We also analysed the disease stage among family contacts and index patients to investigate the role of household contact investigations in diagnosing TB at earlier stages.

\section{Subjects and Methods}

In Taiwan all new cases of active TB are recorded in the National Tuberculosis Registry. In this study we extracted medical and laboratory records from the National Tuberculosis Registry from July 1993 through June 1996 to identify index patients examined at one of 29 hospitals in Taipei. Included in the study were 11873 household contacts of 3903 index patients with newly diagnosed 
Table I. Risk of tuberculosis (TB) transmission among household contacts (including children) of family members with pulmonary $\mathrm{TB}$ 1993-1996.

\begin{tabular}{lc} 
No. of index cases & 3903 \\
No. of household contacts & 11873 \\
No. of contacts & \\
received chest X-ray & 4595 (response rate: $38.7 \%$ ) \\
No. cases found & 284 \\
Rate of cases found & $6.2 \%$ \\
\hline
\end{tabular}

active TB. Of these contacts, 4702 (39.6\%) refused to participate and 2576 (21.7\%) were lost to follow up. Consequently, 4595 consented to receive chest X-ray, yielding an overall response rate of $38.7 \%$. TB was diagnosed on the basis of clinical features, chest X-ray, and sputum findings.

Household contact investigations were conducted by 12 full-time public health nurses under the direction of the Taipei Municipal Chronic Disease Hospital. The public health nurses contacted family members of each patient with newly diagnosed active TB by telephone to inform them of the contagiousness of the disease. The importance of contact investigation was explained to the patients or their family members to prepare the family for subsequent interviews and home visits by the public health nurses. The public health nurses visited the index patients' homes to assemble data, including age, sex, presence of symptoms, etc., on household contacts to notify the contacts of their potential exposure to $\mathrm{TB}$ and to impress on the index patients the need to bring household contacts to receive chest X-ray examination. Chest X-ray examination was advised for the adult contacts. Child contacts (less than 15 years of age) received the Mantoux tuberculin skin test; if the results were positive, radiography was recommended. Sputum evaluation was also performed for all contacts with abnormal chest X-ray findings, or on any suspicion of active disease. The extent of the pulmonary disease in index patients and contacts was categorized as minimal, moderately advanced, or far advanced. The extent of the pulmonary disease was classified according to ATS (American Thoracic Society) recommendation in 1950.

\section{Results}

During the 3-year study period, 3903 patients with newly diagnosed active TB were examined at one of the 29 Taipei hospitals included in this study. The index patients had a total of 11873 household contacts. Among these contacts, 4595 consented to receive chest X-ray, for a response rate of $38.7 \%$. Of these, $284(6.7 \%)$ had newly diagnosed active pulmonary TB. The extent of the disease was minimal in $188(66.3 \%)$ of family contacts with $\mathrm{TB}$,
Table II. Classification of the pulmonary tuberculosis (TB) among index patients and household contact, 1993-1996.

\begin{tabular}{lll}
\hline $\begin{array}{l}\text { Extent of } \\
\text { disease }\end{array}$ & $\begin{array}{l}\text { Household contacts } \\
\text { with active TB }(n=284) \\
\text { Number (per cent) }\end{array}$ & $\begin{array}{l}\text { Index patients } \\
\text { with active TB }(n=3903) \\
\text { Number (per cent) }\end{array}$ \\
\hline $\begin{array}{l}\text { Minimal } \\
\begin{array}{l}\text { Moderately } \\
\text { advanced }\end{array}\end{array}$ & $\begin{array}{l}79(27.8) \\
\text { Far advanced }\end{array}$ & $1261(32.3)$ \\
\hline
\end{tabular}

moderately advanced in $79(27.8 \%)$, and far advanced in only 17 (5.9\%). By contrast, only 32.3\% (1261) of the 3903 index patients had minimal disease, while 51.8\% (2022) had moderately advanced disease and 15.9\% (620) had far advanced disease.

\section{Discussion}

The results of this study show that the risk of TB infection among household contacts of index TB patients in Taipei is high and contact studies can identify large numbers of new TB cases. Households of persons with active TB serve as breeding places for $\mathrm{TB}$, and household contact investigation has proved very efficient in finding TB cases.

Several studies ${ }^{6-9}$ have suggested that household contact investigation is an important epidemiological tool for uncovering cases of active TB in the community. In 1962 the value of family contact investigation was borne out by a nationwide study of the U.S. Public Health Service, ${ }^{8}$ which identified 479 new cases of active TB in an examination of 25512 household contacts $(1.9 \%) .{ }^{8}$ In the present study, investigation of 4595 household contacts of 1436 index TB patients identified $284(6.2 \%)$ new cases of active pulmonary TB. This rate of new cases is about 15 times higher than that reported in a mass chest X-ray survey of the general population in Taipei $(0.42 \%) .{ }^{10}$

Several factors could explain this unusually high case yield. One is that sharing the same indoor environment with a source patient for prolonged periods increases the probability of TB infection. However, it should be emphasized that virtually all household contacts found to have active primary TB in this study were apparently healthy at the time of diagnosis: they were unaware that anything was wrong until the chest X-ray revealed active TB. This stresses the importance of chest radiography and warns against the illusion that family contacts with ostensibly good health do not need medical attention. Struthers and colleagues $^{7}$ suggested that intensive contact investigation permits early diagnosis, and such an approach would greatly reduce the number of new cases and hasten control of the disease. Their study of TB contacts in Seoul, 
Korea, in 1959 showed that the extent of disease among family contacts is especially noteworthy. In their study, $65 \%$ of the family contacts with TB had minimal disease, while $26.3 \%$ had moderately advanced disease and only $8.7 \%$ had far advanced disease. Thus, the majority of the cases were discovered in the early stages of the disease when the therapeutic success rate is the highest and the public health hazard is minimal. Our study showed similar findings, in that family contacts, overall, had less advanced disease than did index patients: roughly twothirds of the family contacts with TB had minimal disease, while two-thirds of the index TB patients had moderately advanced or far advanced disease. These findings further support the value of household contact investigation for diagnosing TB at earlier stages, when chemotherapy can produce more favourable results.

It is apparent that in the past many household contacts were never seen at all, or were seen only when serious symptoms arose. With the increased mobile $\mathrm{X}$-ray vehicles available in Taiwan, it is now feasible to obtain roentgenograms of all household contacts. The dedicated contact-tracing service achieved a response of only $38.7 \%$ in the present study, so by extrapolation from the 284 cases found a further 426 were missed - clearly a serious public health concern even in the context of the high number of cases identified annually in Taiwan. It is important to assess what might be the barriers to people accepting screening in order that the resource of a tuberculosis charge-free service may be directed in the most profitable way. Several factors could explain this low response rate. One is that the household contacts were unaware that anything was wrong when the nurses visited home. Tyrrell et al. ${ }^{11}$ in a special investigation in Glasgow, succeeded in examining 413 of 415 family contacts, and thus showed that almost complete acceptance of contact examination could be achieved. The secret of their success seemed to have been the personal approach by the physician himself, including visits to the household.

In summary, our data show a high risk of infection among family members of patients with active TB. The results of the study also show that household contact investigation is an efficient method for discovering large numbers of TB cases, and may have a remarkable impact on the early diagnosis of pulmonary TB. A dural approach is necessary by which cases are traced from adult patients to child contacts, and from child patients to adult contacts. Widespread application of this approach may help achieve the goal of TB control in Taiwan.

\section{Acknowledgment}

The authors are grateful to the 12 public health nurses of Taipei Municipal Chronic Disease Hospital for collecting all the home-visit data and information that make this study possible.

\section{References}

1 Kline SE, Hedemark LL, Davies SF. Outbreak of tuberculosis among regular patrons of a neighborhood bar. $N$ Engl J Med 1995; 333: 222-227.

2 Beck-Sague C, Dooley SW, Hutton MD et al. Hospital outbreak of multidrug-resistant. M. tuberculosis infection. JAMA 1992; 268: 1280-1286.

3 Houk V, Baker J, Sorensen K, Kent DC. The epidemiology of tuberculosis infection in a closed environment. Arch Environ Health 1968; 16: 26-35.

4 Kenyon TA, Valway SE, Ihle WW et al. Transmission of multidrugresistant Mycobacterium tuberculosis during a long airplane flight. N Engl J Med 1996; 334: 933-938.

5 The Lodi Tuberculosis Working Group. A school and communitybased outbreak of M. tuberculosis in northern Italy. Epidemiol Infect 1972; 113: 83-93.

6 Sue K H-K. Contact investigation: a practical approach to tuberculosis eradication. AJPH 1963; 53: 1761-1769.

7 Struthers EB, Lee HK, Ham SS et al. Tuberculosis contacts in Seoul, Korea. Am Rev Resp Dis 1961; 93: 808-813.

8 Ferebee SH, Mount FW. Tuberculosis morbidity in a controlled trial of the prophylactic use of isoniazid among household contacts. Am Rev Resp Dis 1962; 85: 490-510.

9 Rose CE, Zerbe GO, Lantz SO, Bailey WC. Establishing priority during investigation of tuberculosis contacts. Am Rev Resp Dis 1979; 119: 603-609.

10 Department of Health: The eighth survey report of tuberculosis prevalence in Taiwan. Department of Health, Executive Yuan 1993.

11 Tyrrell WF and Smith J. Pulmonary tuberculosis in North Glasgow. BMJ 1956; 5007: 1451. 